\title{
Treatment of idiopathic inflammatory orbital pseudotumours by radiotherapy
}

\author{
J. C. ORCUTT,${ }^{1 *}$ A. GARNER,${ }^{2}$ J. M. HENK,${ }^{3}$ AND J. E. WRIGHT ${ }^{1}$ \\ From the 1 Orbital Clinic, Moorfields Eye Hospital, City Road, London; the ${ }^{2}$ Institute of Ophthalmology, \\ Cayton Street, London; and the ${ }^{3}$ Royal Marsden Hospital, Fulham Road, London
}

SUMMARY Radiotherapy offers an alternative to steroid therapy for idiopathic inflammatory orbital pseudotumours. Twenty-four orbits were treated with radiotherapy, of which $75 \%$ responded adequately. There were no significant complications and no recurrences during the average follow-up time of 22.3 months (1-50 months). Biopsy, CT scanning, and steroid response of orbital pseudotumours can be helpful in predicting the response to radiotherapy. An adequate response to radiotherapy can be predicted if lymphocytes with germinal centres are present in biopsy specimens. Polymorphonuclear leucocytes and eosinophils suggest a poor response to radiotherapy. Small, discrete, inflammatory masses respond better than large diffuse masses. Pseudotumours initially responding to steroids were likely to respond to radiotherapy.

Orbital pseudotumours are inflammatory lesions of unknown aetiology which simulate neoplasms. They commonly present with pain and lid or conjunctival oedema. Ophthalmoplegia, proptosis, displacement, and decreased visual acuity may also be presenting signs. ${ }^{1-4}$ Commonly biopsy is required to exclude malignancy.

Idiopathic inflammatory orbital pseudotumours are usually treated with systemic steroids. ${ }^{135} 25$ to $50 \%$ of patients do not respond to steroids, ${ }^{15}$ and long-term therapy may lead to serious complications, which can be life-threatening. Radiotherapy can be used to treat these lesions. ${ }^{16-10}$

This report describes the treatment of patients with idiopathic inflammatory orbital pseudotumour with supervoltage radiotherapy. The radiotherapy response has been correlated with biopsy results, CT scan results, and initial steroid response in order to derive predictive criteria for response to therapy.

\section{Patients and methods}

Twenty-two patients fulfilled the criteria for idiopathic inflammatory orbital pseudotumour and were treated with radiotherapy between 1971 and 1981.

Correspondence to Mr John E. Wright, FRCS, Moorfields Eye Hospital, City Road, London EC1V 2PD.

* Present address: Department of Ophthalmology, University of Washington, RJ-10, Seattle, Washington 98195, USA.
Other causes of orbital inflammation were excluded. Patients with possible Graves's ophthalmopathy underwent biochemical tests for thyroid function, namely, T3, T4, thyrotropin-releasing hormone (TRH) and thyroid stimulating hormone (TSH). Radiographs of the skull were taken, including sinus and optic foramen views, together with orbital ultrasonography. Nineteen patients had orbital CT scanning.

The clinical diagnosis was confirmed in 20 patients by orbital biopsy. A chronic, polymorphic inflammatory response was present in each case. Patients with lymphoma or a specific inflammatory process were eliminated from the study. One patient did not have a biopsy done because no mass was seen on CT scan, and one patient's biopsy was not available for review. The bilateral cases underwent biopsy on the side presenting initially.

$X$-rays and CT scans of these patient were evaluated for mass size, myositis, location of mass, morphology, and contiguous sinusitis. The tissue samples were reviewed by an independent observer without prior reference to the original pathological diagnosis or respone to treatment. Each histological section was evaluated for the presence of lymphocytes (including immature, nonfollicular, blast forms), germinal centres, plasma cells, eosinophils, macrophages, polymorphonuclear neutrophils $(\mathrm{PMN})$, and fibrosis. 
RADIOTHERAPY

Twenty-four orbits were treated by radiotherapy. It was administered with the aim of delivering a tumour dose of 2500 rads ( 25 gray) in 12 fractions, the treatment being for 5 days per week, so that the overall treatment time was usually 16 days. The radiotherapy technique employed depended on the site and extent of the lesion as demonstrated by clinical examination and CT scanning. One patient whose lesion was situated anteriorly was treated by single direct anterior field with $250 \mathrm{kV} x$-rays, the cornea being shielded with a $1.5 \mathrm{~cm}$ diameter lead disc suspended in the beam. All the other patients in this report were treated using a cobalt- 60 beam unit operating at $70 \mathrm{~cm}$ source-skin distance. Extraconal masses were irradiated with anterior and lateral wedged fields, the cornea being shielded from the anterior field (Fig. 1). Intraconal lesions were treated either by single lateral fields or by superolateral or inferolateral wedged field.

\section{STEROID THERAPY}

Eighteen of the 22 patients (19 orbits) had received prednisolone prior to radiotherapy. The dosage ranged between 20 and $80 \mathrm{mg}$ daily. The 4 patients who did not receive steroids included 2 who preferred radiotherapy over the potential side effects of steroids and 2 foreign patients for whom long-term follow-up on steroids was not possible.

\section{Results}

There were 12 men and 10 women patients. Their ages ranged from 7 to 72 years, average 50 years. The right orbit was involved in 13 cases, the left orbit in 7 , and 2 cases $(9 \%)$ were bilateral. This population was typical for patients with pseudotumour. ${ }^{1-3}$ The most common presenting symptoms were lid or conjunctival oedema (15 orbits) and pain (13 orbits). Decreased visual acuity (1 orbit), proptosis (2 orbits),

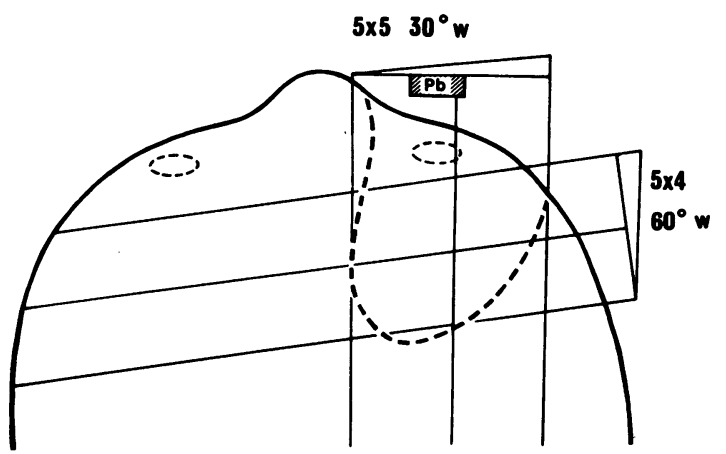

Fig. 1 Radiotherapy fields for treatment of extraconal orbital pseudotumours.
Table 1 Response to radiotherapy

\begin{tabular}{llll}
\hline & Full & Partial & None \\
\hline Orbits & $11(46 \%)$ & $7(29 \%)$ & $6(25 \%)$ \\
Follow-up (months) & $21 \cdot 5$ & $25 \cdot 7$ & $20 \cdot 0$ \\
Range & $(1-50)$ & $(4-38)$ & $(2-35)$ \\
\hline
\end{tabular}

See text for definitions of full and partial radiotherapy response.

and diplopia (4 orbits) were less common. Lid or conjunctival oedema was the most common presenting sign, being present in all 24 orbits. Proptosis was present in 21 orbits. The presenting visual acuity was $6 / 6$ or better in 8 eyes, $6 / 9$ to $6 / 12$ in 8 eyes, $6 / 18$ to $6 / 60$ in 4 eyes, and counting fingers or less in 3 . The visual acuity could not be obtained from one patient due to senility. Ophthalmoplegia was present in 19 orbits.

\section{RADIOTHERAPY}

Response to radiotherapy was divided into 3 categories. Full response describes patients who had complete recovery with no residual signs or symptoms. Partial response describes the patients who showed significant improvement in their presenting complaints without the need for steroids. Residual signs may persist, but for inclusion in this group, the patients had to state they felt the response was adequate. The third group were patients who did not improve with radiotherapy.

Eleven orbits $(46 \%)$ responded fully to radiation. Seven orbits $(29 \%)$ showed a partial response to radiotherapy. The remaining 6 orbits $(25 \%)$ were unchanged following radiotherapy (Table 1). The mean follow-up after onset of symptoms was 37.9 months and after radiation was $22 \cdot 3$ months (1-50 months).

Pseudotumour has not recurred in any of the orbits which initially responded fully to radiotherapy. None of the partial responders had recurrence of their presenting symptoms, and they continued to accept this response as adequate.

There were no sequelae of radiotherapy. One patient had a mild cataract before radiotherapy which progressed over the next 38 months, limiting his acuity to counting fingers. Radiation may have hastened its opacification. Six patients were unresponsive to radiotherapy. Three patients developed vision of hand motion or worse. One patient has persistent proptosis, one persistent pain, and one has a fixed globe with diplopia. Three patients remain on oral steroids.

One patient presented with orbital pseudotumour in 1971. The biopsy disclosed severe lymphocytic infiltration, very occasional blast forms, no follicles, and occasional macrophages. The patient initially 
responded to steroids, but when these were tapered the pseudotumour recurred. Two years later the patient developed orbital pseudotumour in the second eye. Both orbits responded fully to radiotherapy in 1978. The patient presented in January 1982 with lymphoma involving the lumbar spine, and histological examination showed marked lymphocytic proliferation with numerous centroblasts and centrocytes in the absence of germinal centres. The radical difference in histological appearances between the more recent unequivocally malignant lesion and the original relatively inocuous orbital mass is in keeping with the suggestion that initially reactive lymphoproliferative disease may occasionally give rise to overt malignancy. ${ }^{11}$ There was no orbital recurrence.

STEROID THERAPY

Patients were listed as a positive initial responders if their presenting signs initially resolved on steroids. Patients were called nonresponders if their symptoms did not change or worsened on steroids.

Table 2 shows that of the 13 orbits initially responding to steroids 12 subsequently responded either fully or partially to radiotherapy. Two of the 6 orbits which were initially nonsteroid responders subsequently responded partially to radiotherapy.

The primary reasons for discontinuing or not starting steroids were side effects $(38 \%)$. These included cushingoid symptoms and signs, growth retardation, weight gain, risk of development of gastrointestinal bleeding, and inability to obtain follow-up on steroid therapy. The steroid nonresponders constituted $29 \%$ of the total. Patients relapsing with symptoms while tapering off steroids constituted $33 \%$.

\section{HISTOLOGY}

Three of 4 patients whose biopsy specimen showed severe lymphocytic infiltrates responded fully to radiotherapy. The presence of mild to moderate lymphocytic infiltrates was not correlated with radiotherapy response. Follicles were present in 4 biopsies. Two of these patients responded fully to

Table 2 Steroid and radiotherapy response compared

\begin{tabular}{llrrrl}
\hline & & \multicolumn{2}{l}{ Steroid response } \\
\cline { 3 - 5 } & & + & - & $\begin{array}{l}\text { None } \\
\text { given }\end{array}$ \\
\hline Radiotherapy & & & & 5 \\
response & Full & 11 & 8 & 0 & 3 \\
& Partial & 7 & 4 & 2 & 1 \\
& None & 6 & 1 & 4 & 1 \\
\hline
\end{tabular}

See text for definition of steroid and radiotherapy response. $+=$ Positive steroid response. $-=$ No response to steroids.
Table 3 Histological predictors of radiotherapy response

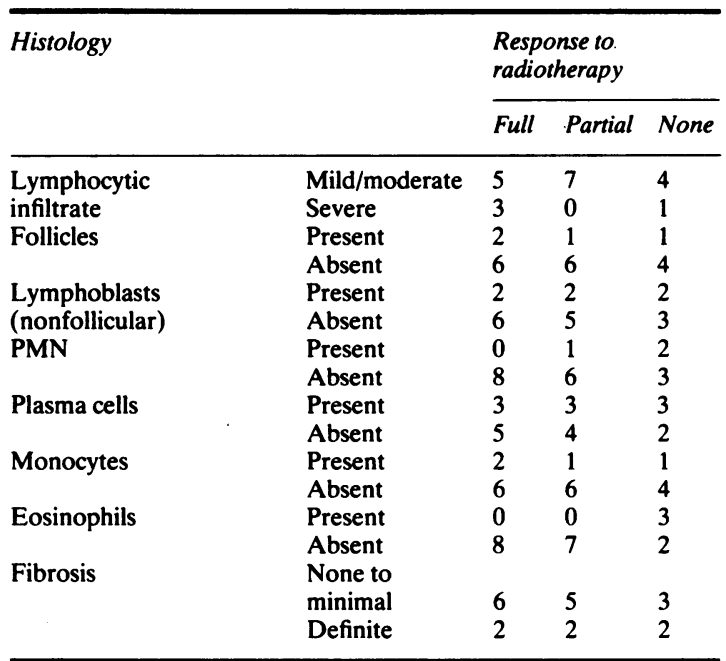

The amount of lymphocytic infiltrate was graded as mild to moderate and severe. The amount of fibrosis was graded as none to minimal and definite. Follicles, polymorphonuclear leucocytes (PMN), plasma cells, monocytes, and eosinophils were noted if present in more than normal numbers. Lymphoblasts were considered present if they were nonfollicular and present in greater than normal numbers.

radiotherapy and one patient responded partially. The absence of follicles did not have any predictive value. Three patients, of whom 2 did not respond to radiotherapy, had PMN in their biopsy specimens. Eosinophils were present in 3 biopsies, and none of these patients responded to radiotherapy. The absence of these cell types did not correlate with the response. The presence or absence of plasma cells, immature nonfollicular lymphocytes, monocytes, or fibrosis was of no value in predicting radiotherapy response (Table 3 ).

\section{RADIOLOGY}

The 4 patients who had small masses and the patient with an extraconal mass responded adequately to radiotherapy (Table 4). The 2 patients who had discrete masses responded fully to radiotherapy (Table 4). Neither myositis nor sinusitis was useful in predicting the outcome of radiotherapy (Table 4).

\section{Discussion}

RESPONSE TO RADIOTHERAPY

Inflammatory orbital pseudotumours adequately responded to radiotherapy in $75 \%$ of the cases. This includes the group that fully responded to radiotherapy (46\%) and the group with partial response $(29 \%)$. Both responses were probably included in the $72 \%$ favourable response reported by Sergott et al. ${ }^{6}$ 
Table 4 Radiological predictors of radiotherapy response

\begin{tabular}{lllll}
\hline Radiology & & \multicolumn{3}{l}{$\begin{array}{l}\text { Response to } \\
\text { radiotherapy }\end{array}$} \\
\cline { 3 - 5 } & & Full & Partial & None \\
\hline Mass & Large & 2 & 4 & 4 \\
Myositis & Small & 3 & 1 & 0 \\
& Present & 4 & 3 & 1 \\
Location & Absent & 3 & 4 & 4 \\
& Intraconal & 3 & 2 & 1 \\
Morphology & Extraconal & 1 & 0 & 0 \\
& Both & 1 & 2 & 4 \\
Sinusitis & Diffuse & 3 & 5 & 4 \\
& Discrete & 2 & 0 & 0 \\
& Present & 2 & 2 & 3 \\
& Absent & 5 & 5 & 2 \\
\hline
\end{tabular}

Small masses were defined as equal to or less than one-half globe diameter in greater dimension. Myositis was present if there was enlargement of one or more muscles on CT scan or orbital ultrasound. The masses with well defined limits were defined as discrete, while those with poorly defined margins were diffuse.

They included both patients who had complete resolution and improvement in their signs and symptoms. A comparison of these groups is difficult in that exact criteria for inclusion in the favourable group were not presented.

The patients who adequately responded to radiotherapy in this study have not suffered recurrence of signs or symptoms for a mean follow-up time of $22 \cdot 3$ months. Response to radiotherapy probably represents a permanent cure. Systemic steroids may be curative but in some cases are palliative in that signs and symptoms recur as the steroid dose is reduced. The average time after radiotherapy for response to occur for the full responders was $3 \cdot 2$ months and for the partial responders was 7.9 months. Nonresponders are unlikely to improve after radiotherapy, since they have been follow up for an average of 20.0 months.

This series suggests that radiotherapy has a place in the management of idiopathic inflammatory orbital pseudotumour, especially in those patients who initially respond well to steroids but relapse. It may be particularly useful as the first line of treatment in those patients with relative medical contraindications to steroid therapy, such as peptic ulceration, especially where histology and radiology predict a good response. The condition is fortunately rare in children and adolescents, but here there is a particular problem. The harmful effects of prolonged steroid therapy, including growth delay, must be weighed against the potential carcinogenic effect of radiation; the latter is probably the lesser risk to these patients.

STEROID RESPONSE

Sergott et al. ${ }^{6}$ concluded that steroid response could not be used as an accurate predictor of radiotherapy response. This was based on 13 orbits, 9 of which responded to steroids and radiotherapy and 4 which responded to steroids but not to radiotherapy. In this series 13 patients initially responded to steroids and 12 of these subsequently responded either fully or partially to radiotherapy. Of the 6 nonsteroid responders 2 subsequently responded partially and 4 did not respond to radiotherapy (Table 2). This is contrary to the findings of Sergott et al., ${ }^{6}$ who reported 2 patients unresponsive to steroids who fully responded to radiotherapy. Nevertheless it is our opinion that the response to radiotherapy can usually be predicted by the initial steroid response.

\section{HISTOLOGICAL PREDICTORS OF RADIOTHERAPY RESPONSE}

Radiotherapy response was generally adequate if follicles of relatively large number of lymphocytes uncontaminated with other types of leucocytes were present in orbital biopsy. This is the likely reason for the high proportion of patients responding to radiotherapy in previous reports. Kim and Roth ${ }^{8}$ presented 3 patients, 2 of whom showed generalised lymphocytic infiltration. All 3 patients responded to radiotherapy. Donaldson et al. ${ }^{9}$ presented 5 patients with lymphocytic pseudotumours and they all responded to radiotherapy. Kennerdall et al. ${ }^{7}$ biopsied 10 orbital lesions which proved to be due to lymphocytic hyperplasia. All responded to radiotherapy. Sergott et al. ${ }^{6}$ biopsied 13 of 21 orbits. Six orbits were diagnosed as either pseudolymphoma or lymphoid hyperplasia. These terms were not defined but probably implied the presence of numerous lymphocytes. All these cases responded favourably to radiotherapy.

The presence of eosinophils implies that radiotherapy may not be successful. All patients who fully responded to radiotherapy had either no eosinophils or they were only rarely seen. Interestingly, Kim and Roth $^{8}$ had one patient in whom the predominant cellular component was eosinophils. This patient was thought to have necrotising vasculitis and responded well to radiotherapy, in contradiction to the evidence in this report.

The absence or presence of fibrosis on biopsy had no bearing on the radiotherapy response. Sergott $e t$ $a l .6$ had 2 patients with histological evidence of fibrosis. One responded and one did not respond to radiotherapy. This is compatible with the nonpredictive character of fibrosis.

\section{RADIOLOGICAL PREDICTORS OF}

RADIOTHERAPY RESPONSE

Small tumours which were located extraconally and were discrete responders fully or partially to radiotherapy. This is probably a manifestation of inflammatory mass rather than cellular type. There is no 
correlation between the cases with severe lymphocytic infiltration of follicles and the CT appearance. The presence of myositis or sinusitis was of no predictive value.

Pretreatment radiological studies aid in establishing a diagnosis and planning a surgical biopsy approach. In addition, valuable information relating to the prognosis for the response to radiotherapy can be gleaned from these studies.

\section{CONCLUSIONS}

Radiotherapy is an effective treatment for orbital pseudotumour. $75 \%$ of patients adequately responded to it, and the response can be predicted. Biopsy specimens showing severe lymphocytic infiltration with follicles suggests an adequate radiotherapy response. The presence of follicles has been shown to correlate with steroid response or spontaneous remission. ${ }^{5}$ Thus the presence of follicles would imply a good outcome regardless of the type of treatment. An unfavourable response to radiotherapy would be predicted if eosinophils or polymorphonuclear leucocytes were present in the biopsy. Small discrete masses on CT scan respond to radiotherapy better than large diffuse masses. An initial positive response to steroids implies a greater likelihood of response to radiotherapy.
Our thanks are due to Mrs Sarah Cole for typing the manuscript.

\section{References}

1 Hearsink B, Rodrigues MR, Flanagan JC. Inflammatory pseudotumor of the orbit. Ann Ophthalmol 1977; 9: 17-29.

2 Blodi FC, Gass JDM. Inflammatory pseudotumor of the orbit. Trans Am Acad Ophthalmol Otolaryngol 1967; 71: 303-23.

3 Jakobiec FA, Jones IS. Orbital inflammations. In: Duane T, ed. Clinical ophthalmology. Hagerstown: Harper and Row, 1973: 2: chapter 23.

4 Henderson JW, Farrow GM. Orbital tumors. Philadelphia: Saunders, 1973: 597.

5 Chavis RM, Garner A, Wright JE. Inflammatory orbital pseudotumor. Arch Ophthalmol 1978;96: 1817-22.

6 Sergott RC, Glaser JC, Chargulu K. Radiotherapy for idiopathic inflammatory orbital pseudotumor. Arch Ophthalmol 1981; 99: 853-6.

7 Kennerdall JS, Johnson BL, Deutsch M. Radiation treatment of orbital lymphoid hyperplasia. Ophthalmology 1979; 86: 942-7.

$8 \mathrm{Kim}$ RV, Roth RE. Radiotherapy of orbital pseudotumor. Radiology 1978; 127: 507-9.

9 Donaldson SS, McDougall IR, Eghert PR, Enzmann DR, Kriss JP. Treatment of orbital pseudotumor (idiopathic orbital inflammation) by radiation therapy. Int J Radiat Oncol Biol Phys 1980; 6: 79-86.

10 Henk JM. Role of the radiotherapist in orbital disease. Trans Ophthalmol Soc UK 1979; 99: 234-5.

11 Jakobiec FA, Jones IS. Lymphomatous, plasmacytic, histiocytic and hematopoietic tumors. In: Jones IS, Jakobiec FA, eds. Diseases of the orbit. New York: Harper and Row, 1979: 309-53. 